Faculdade de Medicina de Ribeirão Preto

Universidade de São Paulo

Nathalia Cunha Calixto

- Gliomas de vias ópticas e estudo volumétrico por Ressonância Magnética: A quimioterapia importa?

Ribeirão Preto

2016

Nathalia Cunha Calixto 


\section{- Gliomas de vias ópticas e estudo volumétrico por Ressonância Magnética: A quimioterapia importa?}

Dissertação apresentada à Faculdade de Medicina de Ribeirão Preto da Universidade de São Paulo, para obtenção do título de Mestre profissional pelo programa de mestrado profissional em Ciências das Imagens e Física Médica.

Área de Concentração: Diagnóstico por Imagem

Orientador: Gustavo Novelino Simão

Versão corrigida. A versão original encontra-se disponível tanto na Biblioteca da Unidade que aloja o programa, quanto na Biblioteca digital de Teses e Dissertações da USP (BDTD)

\section{Ribeirão Preto}


AUTORIZO A REPRODUÇÃO E DIVULGAÇÃO TOTAL OU PARCIAL DESTE TRABALHO, POR QUALQUER MEIO CONVENCIONAL OU ELETRÔNICO, PARA FINS DE ESTUDO E PESQUISA, DESDE QUE CITADA A FONTE. 
Calixto, Nathalia Cunha.

- Gliomas de vias ópticas e estudo volumétrico por Ressonância Magnética: A quimioterapia importa? / Nathalia Cunha Calixto; Orientador: Gustavo Novelino Simão - Ribeirão Preto, 2016.

30 páginas, 7 ilustrações, 4 tabelas.

Dissertação de mestrado profissional (Programa Ciências das Imagens e Física Médica - Área de Concentração: Diagnóstico por Imagem) - Faculdade de Medicina de Ribeirão Preto da Universidade de São Paulo.

1) Ressonância magnética; 2) Gliomas óptico-quiasmáticos e hipotalâmicos; 3) pediatria 4) quimioterapia; 5) Volumetria; 6) Neurofibromatose tipo -1 


\section{FOLHA DE APROVAÇÃO}

Nathalia Cunha Calixto

- Gliomas de vias ópticas e estudo volumétrico por Ressonância Magnética: A quimioterapia importa?

Dissertação apresentada à Faculdade de Medicina de Ribeirão Preto da Universidade de São Paulo para obtenção do título de mestre profissionalizante. Área de concentração: Diagnóstico por Imagem.

Aprovado em:

Banca examinadora:

Prof. Dr.

Instituição:

Assinatura:

Prof. Dr.

Instituição:

Assinatura:

Prof. Dr.

Instituição:

Assinatura: 


\section{Agradecimentos}

Aos pacientes e suas famílias.

À minha família, especialmente a minha mãe, que sempre me apoiaram.

Aos meu orientadores, pela colaboração neste trabalho. 


\section{Resumo}

Calixto, NC. Gliomas de vias ópticas e estudo volumétrico por Ressonância Magnética: A quimioterapia importa? Dissertação de mestrado profissional. Faculdade de Medicina de Ribeirão Preto, Universidade de São Paulo.

Os gliomas de vias ópticas (GVO) representam 5\% dos tumores cerebrais pediátricos e geralmente aparecem histologicamente como astrocitomas de baixo grau. Por causa do curso imprevisto dos GVO, as opções de tratamento ainda são controversas, envolvendo vigilância, cirurgia, quimioterapia e radioterapia. Neste estudo, realizamos a análise volumétricas de gliomas de vias ópticas envolvendo as regiões óptico-quiasmáticas e hipotalâmica (GOQH) para comparar a evolução as neoplasias tratadas com e sem quimioterapia, comparando o volume e componentes das lesões.

Foram analisados retrospectivamente 14 pacientes com $(\mathrm{GOQH})$ que foram submetidos a Ressonância Magnética em nosso departamento de janeiro de 2000 a outubro de 2015. Um total de 45 RM de encéfalo foram incluídas, com uma média de 3,2 estudos/paciente. A avaliação das lesões foi realizada manualmente por um Neurorradiologista, usando o Software DISPLAY. Quatro destes pacientes eram portadores de NF-1. Oito foram tratados com quimioterapia, sendo carboplatina e vincristina (Carbo/VCR) os agentes de primeira linha. As medidas volumétricas foram realizadas com separação entre os componentes sólidos e císticos das neoplasia, usando as sequências FLAIR e T1 pós contraste, com o apoio de imagens ponderadas em $\mathrm{T} 1$ e $\mathrm{T} 2$.

Um aumento de aproximadamente $30 \%$ do volume para as lesões sólidas e uma redução de 19,4\% no volume das lesões sólido-císticas foram observados no período global após o tratamento com quimioterapia, porém ambos sem significância estatística. Entre os pacientes não tratados, observou-se uma redução de 16,6 \% do volume global das lesões durante o período de acompanhamento.

A avaliação da eficácia do tratamento para pacientes com GOQH é difícil, dada a raridade de casos e heterogeneidade radiológica. Os dados de algumas publicações argumentam que o valor da quimioterapia é controverso e não se correlaciona com a resposta radiológica. Em nosso estudo observamos uma pequena redução do volume de neoplasias entre os pacientes tratados e não tratados com quimioterapia, porém sem significância estatística. Ensaios clínicos prospectivos são necessários para melhor avaliar o efeito da quimioterapia sobre OPG.

Palavras-chave: quimioterapia; neurofibromatose I (NF1); gliomas óptico-quiasmáticos e hipotalâmicos $(\mathrm{GOQH})$; pediatria, Ressonância Magnética, avaliação volumétrica 


\begin{abstract}
Calixto, NC. Optic Pathway Gliomas and Volumetric MR study: Does the chemotherapy

work?. 2016. Master's Degree (or Thesis). Faculty of Medicine of Ribeirão Preto, University of São Paulo.
\end{abstract}

Optic pathway gliomas (OPG) represent $5 \%$ of pediatric brain tumors and generally appear histologically as low-grade astrocytomas. Because of the unpredictable course of OPG, adequate treatment method has been controversial, involving surveillance, surgery, chemotherapy and radiotherapy. In this study, we use volumetric imaging to compare evolution between OPG treated with and without chemotherapy, analyzing the volume and components of the lesions.

We retrospectively analyzed 14 patients with OPG who underwent MRI in our department from January 2000 to October 2015. A total of 45 brain MRI were included, with an average of 3,2 studies/patient. The assessment of lesions was manually performed by a neuroradiologist, using software DISPLAY. Four of these patients had NF-1. Eight were treated with chemotherapy, using carboplatin and vincristine (Carbo/VCR) as first-line agents. Volumetric measurements of tumors were segmented into solid and cystic components using FLAIR and T1 weighted images after Gadolinium sequences, with support of T1 and T2 weighted images.

An increase of approximately $30 \%$ of volume for solid lesions and a decrease of $19,4 \%$ for solid-cystic lesions were noted following chemotherapy in overall period, both with no statistical significance. Among patients not treated with chemotherapy, we observed a reduction of $16 \%$ in overall volume of the lesions

Evaluation of treatment efficacy for OPG patients is difficult, given the rarity of cases and radiological heterogeneity. Data from some publications argued that the value of chemotherapy is controversial and does not correlate with radiological response. From our study we observed a small volume reduction of neoplasms among patients treated and not treated with chemotherapy. Larger prospective clinical trials are needed to better evaluate the effect of chemotherapy on OPG.

Keywords: chemotherapy; neurofibromatosis I (NF1); optic chiasmatic-hypothalamic gliomas (OCHG); pediatric, Magnetic Ressonance Imaging, volumetric assessment 


\section{Lista de abreviaturas e siglas}

- GOQH: Gliomas óptico-quiasmáticos e hipotalâmicos

- RM: Ressonância magnética

- NF-1: Neurofibromatose 1

- USP: Universidade de São Paulo

- Carbo/VCR: Carboplatina e Vincristina

- WHO: World Health Organization 


\section{Lista de Tabelas}

Tabela 1: Características demográficas do grupo 1

Tabela 2: Características demográficas do grupo 1

Tabela 3: Variação da volumetria no Grupo 1

Tabela 4: Variação da volumetria no Grupo 2 


\section{Lista de Figuras}

Figura 1: Exemplo do uso do software Display para realização da volumetria

Figura 2: Variação volumétrica do componente sólido das lesòes

Figura 3: Variação volumétrica do componente cístico das lesões

Figura 4: Exemplo de lesão expasniva da região quiaática-hipotalâmica, na sequência FLAIR

Figura 5: Exemplo de lesão expasniva da região quiaática-hipotalâmica, na sequência T1 póscontraste

Figura 6: Variação volumétrica das lesões do Grupo 1

Figura 7: Variação volumétrica das lesões do Grupo 2 


\section{Sumário}

1. Introdução 12

2. Objetivo 14

2.1 Objetivo principal

2.2 Objetivos específicos

3. Materiais e Métodos

$\begin{array}{lr}\text { 4. Resultados } & 18\end{array}$

5. Discussão 20

6. Conclusão 23

7. Tabelas e Figuras $\quad 24$

$\begin{array}{lr}\text { 8. Referências bibliográficas } & 29\end{array}$ 


\section{Introdução}

Os gliomas de vias ópticas ocorrem principalmente durante a primeira década de vida e representam cerca de 5\% dos tumores cerebrais nesta população [1,2].

Estas lesões podem crescer em qualquer lugar ao longo da via óptica, desde os nervos oculares até o córtex occipital, incluindo as lesões da região óptico-quiasmática e hipotalâmica (GOQH) [3].

Os gliomas de vias ópticas geralmente têm aspecto de imagem bastante característicos na ressonância magnética $(\mathrm{RM})$ e a necessidade de biópsia para definição está atualmente limitada a casos com achados clínicos ou de imagem incomuns. A grande maioria dos gliomas de vias ópticas são patologicamente lesões de baixo grau, muitos sendo astrocitoma pilocítico [4]. A associação com neurofibromatose tipo 1 (NF-1) é importante e bem conhecido na literatura $[2,4,6]$, com prevalência variando de 15-21\% [4]. A apresentação clínica mais comum dos gliomas de vias ópticas, quando sintomáticos, é a diminuição da acuidade visual. Função pupilar anormal, diminuição da visão de cores, atrofia do nervo óptico ou proptose também são comuns [5]. Lesões da região óptico-quiasmática e hipotalâmica $(\mathrm{GOQH})$ podem ainda apresentar distúrbios endocrinológicos, como puberdade precoce e hidrocefalia [7]. Alguns autores relacionaram os GOQH a um pior prognóstico, com uma maior taxa de morbidade em relação aos gliomas da região anterior [3,4,8,9 ].

A classificação mais utilizada para os gliomas de vias ópticas ainda hoje é a Classificação proposta por Dodge (1958) [25] que resumidamente separa as neoplasias entre lesões restritas ao(s) nervo(s) óptico(s) (Estágio I), lesões com extensão ao quiasma óptico com ou sem envolvimento do nervo óptico (Estágio II) e lesões com envolvimento da região hipotalâmica ou de estruturas adjacentes (Estádio III).

Embora os gliomas de vias ópticas já tenham sido historicamente considerados como lesões hamartomatosos benignas e auto-limitadas [10], atualmente é mais aceito que estas lesões sejam neoplasias verdadeiras, com padrões variados de crescimento e evolução clínica imprevisível, variando desde pequenas lesões assintomáticas à tumores localmente agressivos e infiltrativos. Não existem características clínicas ou de neuroimagem específicas para caracterizar os gliomas de vias ópticas agressivos ou indolentes [3].

Por causa destes evolução variável, o método de tratamento é ainda bastante controverso na literatura, incluindo observação, cirurgia, quimioterapia e radioterapia $[11,12,13,14,15]$.

Uma vez que os gliomas de vias ópticas em geral, especialmente os GOQH, não são passíveis de ressecção completa e devido aos riscos inerentes do tratamento radioterápico, a quimioterapia tem sido considerada como o tratamento de primeira linha $[14,16,17,18,19]$. O regime de quimioterapia mais amplamente utilizado deriva dos trabalhos publicados por Packer et al.[14,16], utilizando carboplatina e vincristina (carbo / VCR) em uma fase de indução de dez semanas, seguido por uma fase de 
manutenção de quarenta e oito semanas. Outras opções de tratamento, frequentemente utilizados em caso de alergia à carboplatina ou fracasso do tratamento de primeira linha, são vimblastina semanal [20], e outros regimes multi-agente [21].

O acompanhamento a longo prazo destes pacientes é baseado em avaliações clínicas e neuro-oftalmológicas, bem como em estudos de Ressonância Magnética [22] e o tratamento é geralmente iniciado quando há progressão radiológica ou evidência de deterioração clínica, especialmente comprometimento visual [23].

Neste estudo, foram realizadas medidas volumétricas manuais detalhados das lesões de 14 pacientes portadores de $\mathrm{GOQH}$, comparando as variações de volume e alterações no aspecto de imagem entre tumores tratados e não-tratados com quimioterapia. 


\section{Objetivos}

\subsection{Geral:}

Realizar uma análise volumétrica das lesões de pacientes com gliomas óptico-quiasmáticos hipotalâmicos $(\mathrm{GOQH})$ acompanhados em nossa instituição, comparando a evolução entre os pacientes tratados com quimioterapia e não tratados.

\subsection{Específico:}

Avaliar as mudanças entre os componentes císticos dos GOQHs em relação o à quimioterapia Analisar e comparar os resultados entre pacientes portadores e não portadores de NF-1. 


\section{Materiais e Métodos}

\section{População}

Este estudo foi aprovado pelo comitê de ética da Faculdade de Medicina de Ribeirão Preto da Universidade de São Paulo (HCFMRP-USP), número do processo 46594015.1.0000.5440. Todos os pacientes com diagnóstico de glioma de vias ópticas em acompanhamento na Instituição ao longo dos anos 2001-2015 foram consideradas para este estudo. Os critérios de inclusão foram: presença de lesão expansiva com epicentro na região quiasmática e hipotalâmica (Dodge II / III) [25], idade inferior a 18 anos no momento do diagnóstico, e a presença de pelo menos três estudos de imagem por RM, com pelo menos um exame realizado antes do início do tratamento (baseline). Pacientes com tumores fora da região óptico-quiasmática e hipotalâmica, com tumores muito infiltrativos com baixa reprodutibilidade de avaliação volumétrica e com tumores submetidos a cirurgia durante o follow-up foram excluídos. Vinte e dois pacientes foram inicialmente colocado no estudo. Após avaliação inicial, oito dos pacientes foram considerados ilegíveis por razões diferentes e foram excluídos do estudo.

\section{Diagnóstico e tratamento}

O diagnóstico de GOQHs foi baseado principalmente nos achados de imagem na RM. Dez pacientes também tiveram confirmação histopatológica. Entre os 14 pacientes no estudo, oito pacientes foram tratados com quimioterapia (Grupo 1), com vincristina e carboplatina (VCR / CRB) como agentes de primeira linha em 7 destes, de acordo com o protocolo de Packer $[14,16]$. Cinco destes também foram tratadas com vimblastina semanal, devido a reações adversas ou refratariedade. Um paciente foi tratado com o regime de cisplatina e etoposido como primeira linha.

Seis pacientes (Grupo 2) foram apenas observados durante o intervalo de tempo. 
A avaliação volumétrica das lesões foi realizadas manualmente pelo mesmo neurorradiologista (N.C.C.) para todos os 14 pacientes, usando o software MNI-DISPLAY (Montreal Neurological Institute). A área tumoral foi delineada corte a corte nos exames de Ressonância Magnética e em seguida o volume foi calculado para lesão inteira. A Figura 1 mostra um exemplo da medição volumétrica de lesões utilizando o Software Display. Todos os exames de Ressonância Magnética apresentavam sequências de imagem ponderadas em T2/FLAIR (fluid-attenuation inversion recovery) e sequências ponderadas T1 antes e após a injeção de meio de contraste paramagnético (Gadolínio). Para cada paciente, as medidas de acompanhamento foram obtidas utilizando a mesma sequência de imagem utilizada no exame de Ressonância Magnética inicial.

A avaliação da resposta tumoral foi classificada da seguinte forma: resposta completa, com desaparecimento completo do tumor em exames de imagem; resposta parcial, quando ocorreu uma redução de $50 \%$ ou mais no tamanho do tumor; resposta menor, redução entre 25 a $50 \%$ do volume tumoral; doença estável, sem alteração evidente ou alteração inferior à $25 \%$ do volume da lesão; progressão de doença, aumento igual ou superior a $25 \%$ do volume tumoral $[14,30]$

Para os pacientes tratados com quimioterapia (Grupo 1) analisamos 3 estudos: o primeiro estudo de RM adquirido antes do tratamento (baseline), um estudo imediatamente após o final da quimioterapia (0 - 3 meses depois), considerado como Tempo 2, e um estudo de seguimento tardio após o tratamento com quimioterapia (18 a 24 meses após o fim do tratamento), tempo 3. Para os tumores com componentes císticos e sólidos, aqueles foram medidos separadamente e o volume foi obtido para cada componente.

Para os pacientes que não foram submetidos à quimioterapia analisamos todos os exames de Ressonância Magnética disponíveis no intervalo de tempo. Todos estes tumores tinham componentes exclusivamente sólidos.

A análise estatística foi feita utilizando o teste de Mann-Whitney para amostras independentes. Para foram utilizadas amostras dependentes Wilcoxon pareado. Para determinar a associação entre as variáveis qualitativas, os dados foram submetidos ao teste exato de Fisher (Fisher, 1935). Os dados estatísticos foram posteriormente analisadas com o 
software SAS e R. Todos os testes de significância foram bilaterais; P valores inferiores a 0,05 foram considerados estatisticamente significativos. 


\section{Resultados}

Um total de 14 pacientes foram incluídos no estudo (cinco do sexo masculino e nove do sexo feminino).

Estes pacientes foram divididos em dois grupos: aqueles tratados com quimioterapia (Grupo 1), incluindo oito pacientes (57\%) e aqueles não tratados (Grupo 2), incluindo seis pacientes $(43 \%)$.

Entre as pacientes do Grupo 1 , sete $(87,5 \%)$ foram tratados com vincristina e carboplatina (carbo / VCR) como agentes de primeira linha, de acordo com o protocolo de Packer $[14,16]$. Cinco destes também foram tratadas com vimblastina semanal $(62,5 \%)$ como segunda linha. Um paciente foi tratado com o regime de cisplatina e etoposido como primeira linha (12,5\%). A tabela 1 resume os dados sobre os regimes de tratamento e resultados radiológicos para o Grupo 1.

Dada a associação bem descrita entre NF-1 e gliomas de vias ópticas [2,6], as biópsias não foram realizados em pacientes portadores de NF-1 e achados radiológicos característicos de gliomas de vias ópticas (4 pacientes). Confirmação histopatológica foi obtida para os outros 10 pacientes, sendo 9 submetidos à excisão cirúrgica parcial e um à biópsia da lesão. $\mathrm{O}$ exame histopatológico revelou Astrocitoma Juvenil Pilocítico (AP) em sete amostras, Ganglioglioma (OMS grau 1) em duas amostras e Astrocitoma pilomixóide em uma amostra (OMS grau 2). Para todos os pacientes que foram submetidos a cirurgia ou à biópsia, o estudo por RM definido como baseline foi realizado após os procedimentos.

Quatro pacientes apresentaram tumores com componente cístico. Quatro pacientes eram portadores de NF-1 (28,5\%).

Para o grupo que foi submetido à quimioterapia (Grupo 1), a média de idade no momento do diagnóstico foi de 3,5 anos (variação de 8 meses-11 anos). Para os pacientes que não foram tratados com quimioterapia (Grupo 2) a média de idade média ao diagnóstico foi de 7,4 anos (variação 2-11). Um total de 45 estudos de imagem (RM) foram incluídos. Entre os pacientes com NF-1, apenas um (25\%) foi tratado com quimioterapia.

Efeito da quimioterapia em pacientes OCHGs

A fim de avaliar a resposta ao tratamento de quimioterapia, foi realizada análise volumétrica de tumor antes do tratamento (baseline / Tempo 1), imediatamente após o final da quimioterapia (Tempo 2), e durante acompanhamento tardio (Tempo 3). Para os pacientes que apresentaram lesões sólido-císticas,os volumes foram calculados separadamente para cada componente. As figuras 2 e 3 resumem alterações tumorais para ambos os períodos, dividindo os pacientes entre os pacientes com e sem componente cístico na apresentação.

Analisando os resultados para os tumores exclusivamente sólidos, encontramos um pequeno aumento de volume no tempo $2(5 \%)$ para todo o grupo, seguido por um grande aumento no tempo de $3(30,4 \%)$, porém sem significância significativa ( $p>0,05)$. Não foram encontradas alterações evidentes no aspecto radiológico destas lesões durante o período total de acompanhamento. 
Ao comparar separadamente os componentes sólidos e císticos das lesões, encontramos uma diminuição em ambos os componentes sólidos e císticos para todo o grupo (19,4 e 9,6\%, respectivamente, no período total de avaliação), novamente sem significância estatística ( $\mathrm{p}>$ $0,05)$ e sem alterações evidentes no aspecto de imagem. Um achado interessante é que, para pacientes com lesões sólido-císticas, o componente sólido do tumor parece ter uma melhor resposta ao tratamento quimioterápico $(19,4 \%)$ em relação às lesões exclusivamente sólidas, porém também sem significância estatística.

Quando analisamos a resposta individual ao tratamento para o Grupo 1, nota-se que dois pacientes apresentaram progressão da doença (25\%), dois mantiveram-se estáveis $(25 \%)$ e quatro pacientes $(50 \%)$ experimentaram resposta tumoral, três deles $(75 \%)$ com uma resposta menor e um com resposta parcial (paciente 4).

Embora muitas lesões não apresentaram alterações significativas no aspecto radiológico durante o período de seguimento, muito provavelmente devido seu curso indolente (Fig 4), verificou-se que três pacientes do Grupo 1 apresentaram alterações individuais expressivas. Paciente 2 apresentou um aumento de $50 \%$ no volume da lesão mesmo durante o período de quimioterapia, que permaneceu estável no período de seguimento tardio. Paciente 8 apresentou um aumento de respectivamente, $21 \%$ e de $65 \%$ no volume das lesões nos Tempos 2 e 3, porém este paciente realizou apenas o ciclo de indução do tratamento quimioterápico. Paciente 4 mostrou uma impressionante redução de $70 \%$ do volume da lesão no Tempo 2 com estabilidade em período de seguimento (Figura 5). A Figura 6 mostra alterações individuais no volume de lesões para o grupo 1 .

Avaliação dos pacientes em observação

Para o grupo que não foi submetido à quimioterapia, encontramos ligeira redução do volume tumoral para todo o grupo (17\%) no período de tempo estudado, mas sem significância estatística $(\mathrm{p}>0,05)$.

Quando olhamos para a resposta individual de cada paciente, observou-se que três pacientes (50\%) mantiveram-se estáveis durante o follow-up e os outros três pacientes $(50 \%)$ apresentaram redução parcial espontânea das dimensões da lesão. A Figura 7 mostra a evolução individual dos pacientes do Grupo 2. Os dados referentes aos achados radiológicos no Grupo 2 são apresentados na Tabela 2.

As Tabelas 3 e 4 resumem as mudanças volumétricas para todos os pacientes durante o período de acompanhamento. 


\section{Discussão}

Gliomas de vias ópticas são tumores pediátricos raros com evolução clínica bastante variável, com apresentação desde pequenas lesões assintomáticas encontrados incidentalmente à tumores infiltrativos localmente agressivos.

Apesar de seu comportamento imprevisível, a maioria das lesões são neoplasias de baixo grau, com curso indolente e uma excelente sobrevida global, mas ainda assim podendo apresentar progressão de doença e deficiência visual significativa [28].

Um dos maiores estudos sobre gliomas de vias ópticas na infância foi escrito por Dutton em 1994 [4], com base na revisão de 2.297 casos descritos. A idade média ao diagnóstico foi de 7,0 anos, com acometimento similar entre os sexos masculino e feminino. Segundo esta revisão, a sobrevida média estimada depende da localização do tumor. Quando inicialmente confinado ao nervo óptico, a mortalidade é de cerca de 5\%, mas uma vez que o hipotálamo está envolvido, a mortalidade aumenta acentuadamente a mais de 50\%.

A associação entre gliomas de vias ópticas e NF-1 é amplamente descrita na literatura, com incidência e prevalência destas neoplasias variando segundo à fonte pesquisada, com uma média entre 20 e $40 \%$ na maioria dos estudos [1,4,11,16,20]. Comparado com gliomas esporádicos, gliomas relacionadas à NF1 são menos sintomáticos as diagnóstico e costumam apresentar maior estabilidade ao longo do tempo, com um curso mais indolente $[3,4,6]$.

Devido a sua longa evolução e comportamento errático, o manejo terapêutico destes gliomas ainda é bastante controverso e a recomendação atual é iniciar o tratamento apenas na presença de declínio visual ou progressão radiológica evidente, com uma tendência para a quimioterapia como opção de primeira linha [14,19,23,29 ].

A utilização de métodos de imagem na avaliação destas neoplasias, especialmente a RM, é necessária para a identificação e localização das lesões e também para o monitoramento destas, especialmente na avaliação de progressão da doença e na presença de complicações.

A RM do cérebro e órbitas com cortes finos através dos nervos ópticos e quiasma óptico é a técnica de imagem de escolha para avaliar a extensão destas neoplasias.

Os gliomas de vias ópticas são tipicamente caracterizados pelo aumento difuso dos nervos ópticos e/ou do quiasma, com ou sem extensão à região hipotatâmica ou de regiões posteriores, como tratos e radiações ópticas. Estas lesões são geralmente isointensas nas sequências ponderadas em $\mathrm{T} 1$ e isointensa/hiperintensa nas sequências ponderadas em T2, com realce variável após a administração de contraste. A presença de realce após a administração do gadolínio e a presença de componentes císticos dentro do tumor são mais comuns nos gliomas esporádicos do que em gliomas relacionados à NF1 [5,6,22,24].

Os gliomas de vias ópticas podem ser confinados a áreas específicas da via óptica, como o nervo óptico ou quiasma, ou apresentar uma extensão mais difusa. Quando restrito aos nervos ópticos, geralmente apresentam-se com alargamento circunscrito destes, muitas vezes com uma aparência tortuosa ou em "kinking". Os tumores localizados no quiasma óptico apresentam características mais distintas, variando desde um leve espessamento do quiasma até volumosas lesões suprasselares, com ou sem componente exofítico. Pacientes com gliomas esporádicos tendem à apresentar uma maior proporção de tumores envolvendo o quiasma e vias ópticas posteriores [4, 6, 22]. Por outro lado, pacientes com gliomas relacionados à NF-1 geralmente apresentam lesões localizadas no nervo óptico, que podem ou não podem estender-se ao quiasma. Gliomas bilaterais dos nervos ópticos, sem envolvimento quiasmático, são praticamente diagnósticos de NF-1. 
Apesar de estar em vigor há mais de 20 anos, alguns estudos recentes têm questionado a eficácia da quimioterapia no tratamento de gliomas de vias ópticas, especialmente em relação à melhora da acuidade visual [26,27,31].

Uma recente revisão da literatura [31] incluindo 8 publicações relevantes, não evidenciou melhora visual na maioria das crianças portadoras de gliomas de vias ópticas tratados com quimioterapia, o que está em concordância com alguns outros estudos da literatura [26]. Em uma amostra de 174 pacientes, apenas 25 crianças (14,4\% ) experimentaram uma melhoria na visão, 82 (47,1\%) mantiveram-se estáveis e 67 (38,5\%) sofreram deterioração visual. Em seu estudo multicêntrico de 115 pacientes, Fischer et al. [29] encontraram resultados diferentes, com melhora da acuidade visual identificada em 32\% dos pacientes após a quimioterapia, contradizendo os dados de que a deficiência visual nestes casos é irreversível. Esta série também destaca a dissociação entre os resultados visuais e radiológicos em pacientes portadores de NF-1 com gliomas de vias ópticas tratados com quimioterapia, com apenas 34\% a 38\% de concordância entre os resultados radiológicos e visuais, questionando o uso da ressonância magnética como padrão-ouro de sucesso do tratamento destas neoplasias.

Na prática clínica atual, a avaliação radiológica dos gliomas de vias ópticas é normalmente feita utilizando medidas lineares realizadas em uma única imagem de ressonância magnética e pode ser altamente operador-dependente, com baixa precisão e reprodutibilidade. Considerando esses fatores, fica claro que os métodos de avaliação de resposta radiológicos tradicionais ainda não apresentam sensibilidade suficiente para avaliar com precisão o efeito do tratamento destes gliomas [26].

Em nosso estudo realizamos volumetria detalhada das lesões de quatorze pacientes portadores de lesões da região óptico-quiasmática e hipotalâmica, separando-os em 2 grupos, tratados e não tratados com quimioterapia, em uma tentativa de identificar alterações radiológicas significativas entre esses grupos.

Os resultados encontrados evidenciaram um aumento global de $5 \%$ do volume das lesões para o grupo 1, no tempo 2 e um maior aumento de 30\% no tempo de 3. Para o Grupo 2, encontramos uma ligeira redução $(5 \%)$ do volume total das lesões, ambos sem significância estatística ( $p>0,05)$. Ao analisar a evolução individual destes pacientes, os resultados são mais animadores. Para o Grupo 1 observou-se uma resposta minor ou parcial das lesões em $50 \%$ dos pacientes, estabilidade em $25 \%$ e a progressão de doença em $25 \%$ destes. No Grupo 2 , três pacientes permaneceram estáveis e três apresentaram regressão espontânea parcial de suas dimensões.

Em relação à avaliação das lesões sólido-císticas, foi realizada análise dos componentes sólido e cístico isoladamente, não se observando mudança significativa em seus volumes, em relação ao tratamento. No entanto, vale destacar que as lesões de aspecto sólido-cístico apresentaram uma redução maior do volume do componente sólido com o tratamento quimioterápico, em relação à lesões exclusivamente sólidas. Este achado curioso também foi identificado em outro estudo recente, de Shofty [27].

Existem pouco estudos disponíveis na literatura correlacionando volumetria por RM de gliomas de vias ópticas e uso de quimioterapia. Shofty et al. [27] realizaram estudo volumétrico seguindo algoritmo próprio, de 15 pacientes portadores de neoplasia de vias ópticas e tratados com quimioterapia, encontrando um aumento médio de aproximadamente $12 \%$ do volume dos componentes sólidos em relação ao período estudado. Este resultado está em concordância com os nossos achados.

Em relação ao presente estudo, pode-se observar que em ambos os grupos, a maioria dos pacientes apresentaram uma resposta parcial/menor ou estabilidade das lesões. Este achado é 
provavelmente relacionada ao curso indolente e de baixa agressividade das lesões, e também foi identificado em outros estudos [14,27,32].

Dois pacientes do Grupo 1 apresentaram doença progressiva após o tratamento quimioterápico. Paciente 2 apresentou um aumento de $60 \%$ do volume da lesão no tempo 2 e de $50 \%$ no tempo de 3, após o primeiro (carbo / VCR) e segundo (vimblastina) esquemas de tratamento. A análise individual do paciente 8 evidenciou um aumento de volume de $65 \%$ no período total, seguida de deterioração clínica. Curiosamente, apesar da evolução desfavorável, este foi o único paciente portador de NF-1 da amostra submetido à quimioterapia. No entanto, por razões pouco claras, este paciente recebeu apenas o ciclo de indução do tratamento.

É importante destacar as limitações do nosso estudo, devido à pequena e limitada amostra de pacientes e a não uniformidade entre os protocolos de quimioterapia. Além disso, o provável viés em relação ao agrupamento dos pacientes é outro problema, uma vez que os pacientes submetidos à quimioterapia eram mais jovens, com lesões mais volumosas e sintomáticas, e comportamento possivelmente mais agressivo. Outra limitação é a predominância de NF-1 em pacientes no grupo não tratado, o que pode estar relacionado ao curso mais indolente neste contexto. Além destas, a falta de correlação entre dados clínicos e avaliação visual, restringe a avaliação do tumor apenas aos seus aspectos radiológicos.

Apesar de descritas há mais de 200 anos, os gliomas de vias ópticas ainda são neoplasias desafiadores e com manejo controverso. Métodos de avaliação radiológica mais precisos e reprodutíveis podem ter grande valia na identificação de mudanças sutis destas lesões em relação ao tratamento, melhorando a correlação entre os resultados visuais e radiológicos. 


\section{Conclusões}

Devido ao curso clínico variável e imprevisível, o manejo terapêutico dos gliomas de vias ópticas ainda é bastante controverso. Em nossa pequena e limitada série, não foram observadas diferenças significativas em relação à variação do volume global das lesões tratadas ou não tratados com quimioterapia no período global. A maioria delas se mantiveram estáveis ou apresentaram discreta resposta parcial durante o follow-up, provavelmente refletindo o curso indolente da maioria dos OPGs . 


\section{Tabelas}

Group 1

\begin{tabular}{|c|c|c|c|c|c|c|c|c|c|}
\hline Cases & Sex & $\begin{array}{l}\text { Age at } \\
\text { diagnosis }\end{array}$ & $\begin{array}{l}\text { Surgery/ } \\
\text { Year }\end{array}$ & $\begin{array}{l}\text { Histopathol } \\
\text { ogy }\end{array}$ & $\begin{array}{l}\text { First line } \\
\text { treatment }\end{array}$ & $\begin{array}{l}\text { Second } \\
\text { line } \\
\text { treatment }\end{array}$ & NF-1 & $\begin{array}{l}\text { Follow- } \\
\text { up period } \\
\text { (years) }\end{array}$ & $\begin{array}{l}\text { Overall } \\
\text { Radiological } \\
\text { Changes }\end{array}$ \\
\hline 1 & $\mathrm{~F}$ & 5 years & PE, 2008 & JPA & Carbo/VCR & NA & No & $\begin{array}{l}2011- \\
2015\end{array}$ & MR \\
\hline 2 & $M$ & 3 years & PE, 2008 & JPA & Carbo/VCR & Vinblastine & No & $\begin{array}{l}2010-201 \\
5\end{array}$ & Progression \\
\hline 3 & $\mathrm{~F}$ & 1 year & PE, 1998 & JPA & Carbo/VCR & Vinblastine & No & $\begin{array}{l}2001-201 \\
3\end{array}$ & Stable \\
\hline 4 & $\mathrm{~F}$ & 5 years & PE, 2011 & JPA & Carbo/VCR & Vinblastine & No & $\begin{array}{l}2011-201 \\
5\end{array}$ & PR \\
\hline 5 & $M$ & 1 year & PE, 2010 & JPA & Carbo/VCR & Vinblastine & No & $\begin{array}{l}2010-201 \\
3\end{array}$ & MR \\
\hline 6 & $\mathrm{~F}$ & 8 months & PE, 2013 & $\begin{array}{l}\text { PMA, grade } \\
2\end{array}$ & Carbo/VCR & Vinblastine & No & $\begin{array}{l}2013-201 \\
5\end{array}$ & Stable \\
\hline 7 & $\mathrm{~F}$ & 11 years & PE, 2001 & JPA & $\begin{array}{l}\text { Cisplatin/ } \\
\text { Etoposide }\end{array}$ & NA & No & $\begin{array}{l}2007-201 \\
5\end{array}$ & MR \\
\hline 8 & $\mathrm{~F}$ & 2 years & NA & NA & Carbo/VCR & NA & Yes & $\begin{array}{l}2006- \\
2015\end{array}$ & Progression \\
\hline
\end{tabular}

Table 1: $M=$ Male, $F=$ Female, $P E=$ Partial excision, $J P A=$ Juvenile Pilocytic Astrocytoma, $M R=$ Minor Response, $N A=$ No available, $P M A=$ pilomyxoid astrocytoma, $P R=$ Partial Response, Carbo/VCR: carboplatine/vincristine, NF-1 = Neurofibromatosis,

Group 2

\begin{tabular}{|c|l|l|l|l|l|l|l|l|}
\hline Case & Sex & $\begin{array}{l}\text { Age at } \\
\text { diagnosis }\end{array}$ & Surgery/year & $\begin{array}{l}\text { Histopatholo } \\
\text { gy }\end{array}$ & $\begin{array}{l}\text { Indications } \\
\text { for } \\
\text { treatment }\end{array}$ & $\begin{array}{l}\text { Follow-up } \\
\text { period (years) }\end{array}$ & NF-1 & $\begin{array}{l}\text { Overall } \\
\text { Radiological } \\
\text { Changes }\end{array}$ \\
\hline $\mathbf{1}$ & M & 7 years & PE, 1995/1999 & JPA & Surveillance & $2006-2014$ & No & Progression \\
\hline $\mathbf{2}$ & M & 7 years & NA & NA & Surveillance & $2006-2015$ & Yes & MR \\
\hline $\mathbf{3}$ & F & 2 years & NA & NA & Surveillance & $2011-2014$ & Yes & PR \\
\hline $\mathbf{4}$ & F & 11 years & Biopsy, 2012 & Gangliogioma & Surveillance & $2011-2014$ & No & Stable \\
\hline $\mathbf{5}$ & M & 11 years & NA & NA & Surveillance & $2011-2015$ & Yes & Stable \\
\hline $\mathbf{6}$ & F & 6 years & PE, 1998 & Gangliogioma & Surveillance & $2001-2015$ & No & PR \\
\hline
\end{tabular}

Table 2: $M=$ Male, $F=$ Female, $M R=$ Minor response, $N A=$ No available, NF-1= Neurofibromatosis, $P A$ = Pilocytic Astrocytoma, $P E=$ Partial excision, $P R=$ Partial response 


\begin{tabular}{|c|c|c|c|}
\hline Cases & Baseline & Time 2 & Time 3 \\
\hline 1 & $\begin{array}{l}\text { Solid: } 11,69 \mathrm{~cm} 3 \\
\text { Cystic: } 3,51 \mathrm{~cm} 3 \\
\text { Total: } 15,2 \mathrm{~cm} 3\end{array}$ & $\begin{array}{l}\text { Solid } 1,0 \mathrm{~cm} 3 \\
\text { Cystic: } 3,05 \mathrm{~cm} 3 \\
\text { Total: } 4,05 \mathrm{~cm} 3\end{array}$ & $\begin{array}{l}\text { Solid: } 1,35 \mathrm{~cm} 3 \\
\text { Cystic } 9,04 \mathrm{~cm} 3 \\
\text { Total: } 10,39 \mathrm{~cm} 3\end{array}$ \\
\hline 2 & Solid: 46,2 cm3 & Solid: $70,4 \mathrm{~cm} 3$ & Solid: $74,37 \mathrm{~cm} 3$ \\
\hline 3 & $\begin{array}{l}\text { Cystic: } 5,3 \mathrm{~cm} 3 \\
\text { Total: } 51,5 \mathrm{~cm} 3 \\
\text { Total: } 32,81 \mathrm{~cm} 3\end{array}$ & $\begin{array}{l}\text { Cystic: } 12,22 \mathrm{~cm} 3 \\
\text { Total: } 82,62 \mathrm{~cm} 3 \\
\text { Total: } 30,8 \mathrm{~cm} 3\end{array}$ & $\begin{array}{l}\text { Cystic: } 3,02 \mathrm{~cm} 3 \\
\text { Total: } 77,39 \mathrm{~cm} 3 \\
\text { Total: } 26,6 \mathrm{~cm} 3\end{array}$ \\
\hline 4 & $\begin{array}{l}\text { Solid: } 64,62 \mathrm{~cm} 3 \\
\text { Cystic: } 8,66 \mathrm{~cm} 3 \\
\text { Total: } 73,28 \mathrm{~cm} 3\end{array}$ & $\begin{array}{l}\text { Solid: } 18,43 \mathrm{~cm} 3 \\
\text { Cystic: } 13,56 \mathrm{~cm} 3 \\
\text { Total: } 31,99 \mathrm{~cm} 3\end{array}$ & $\begin{array}{l}\text { Solid: } 19,17 \mathrm{~m} 3 \\
\text { Cystic: } 3,72 \mathrm{~cm} 3 \\
\text { Total: } 22,89 \mathrm{~cm} 3\end{array}$ \\
\hline 5 & Total:22,41 cm3 & Total:12,72 cm3 & Total:15,38 cm3 \\
\hline 6 & $\begin{array}{l}\text { Solid: } 19,47 \mathrm{~cm} 3 \\
\text { Cystic: : } 0,78 \mathrm{~cm} 3 \\
\text { Total: } 20,25 \mathrm{~cm} 3\end{array}$ & $\begin{array}{l}\text { Solid: } 20,77 \mathrm{~cm} 3 \\
\text { Cystic: } 0,66 \mathrm{~cm} 3 \\
\text { Total: } 21,42 \mathrm{~cm} 3\end{array}$ & $\begin{array}{l}\text { Solid: } 19,51 \mathrm{~cm} 3 \\
\text { Cystic: } 0,71 \mathrm{~cm} 3 \\
\text { Total: } 20,22 \mathrm{~cm} 3\end{array}$ \\
\hline 7 & $2,1 \mathrm{~cm} 3$ & $2,0 \mathrm{~cm} 3$ & $1,45 \mathrm{~cm} 3$ \\
\hline 8 & $92,77 \mathrm{~cm} 3$ & $112,09 \mathrm{~cm} 3$ & $152,41 \mathrm{~cm} 3$ \\
\hline
\end{tabular}

TABLE 3: VOLUMETRIC DATA OF LESIONS FOR PATIENTS OF GROUP 1, BEFORE (BASELINE) CHEMOTHERAPY AND IN TIMES 2 AND 3.

\begin{tabular}{|c|c|c|c|c|c|}
\hline Cases & Baseline & Follow-up & Follow-up & Follow-up & Follow-up \\
\hline 1 & $1,65 \mathrm{~cm} 3$ & $2,75 \mathrm{~cm} 3$ & $2,25 \mathrm{~cm} 3$ & $2,54 \mathrm{~cm} 3$ & $1,90 \mathrm{~cm} 3$ \\
\hline 2 & $2,17 \mathrm{~cm} 3$ & $1,47 \mathrm{~cm} 3$ & $1,37 \mathrm{~cm} 3$ & $1,16 \mathrm{~cm} 3$ & \\
\hline 3 & $9,93 \mathrm{~cm} 3$ & $4,4 \mathrm{~cm} 3$ & $4,4 \mathrm{~cm} 3$ & & \\
\hline 4 & $25,54 \mathrm{~cm} 3$ & $29,76 \mathrm{~cm} 3$ & $22,04 \mathrm{~cm} 3$ & $25,69 \mathrm{~cm} 3$ & \\
\hline 5 & $1,49 \mathrm{~cm} 3$ & $1,50 \mathrm{~cm} 3$ & & & \\
\hline 6 & $2,27 \mathrm{~cm} 3$ & $1,05 \mathrm{~cm} 3$ & $1,09 \mathrm{~cm} 3$ & & \\
\hline
\end{tabular}

TABLE 4: VOLUMETRIC DATA OF LESIONS FOR PATIENTS OF GROUP 2 FOR OVERALL PERIOD. 


\section{Figuras}

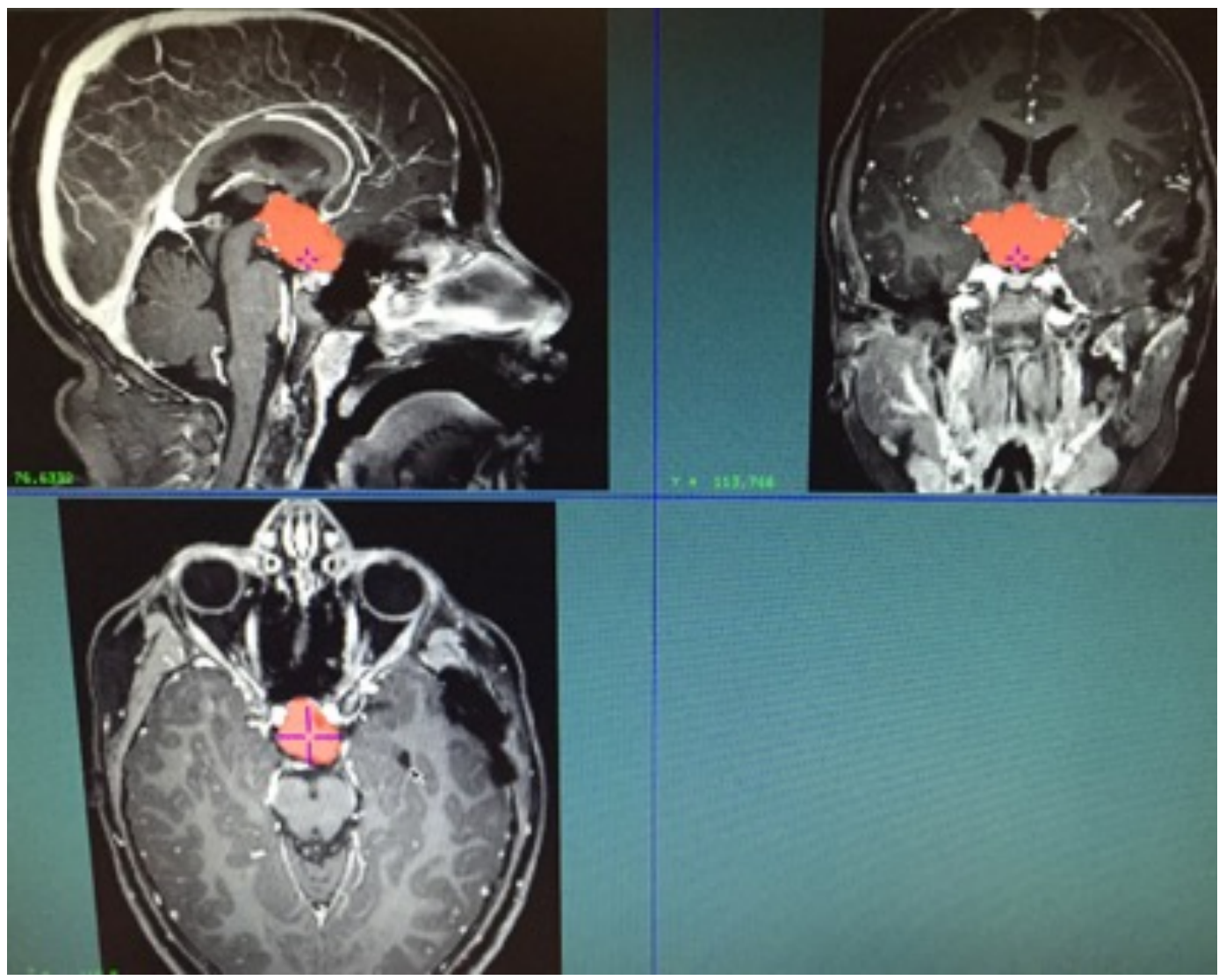

FIG 1: EXEMPLO DA AVALIAÇÃO VOLUMÉTRICA DE LESÃO EXPANSIVA EM REGIÃO QUIASMÁTICA-HIPOTALÂMICA PELO SOFTWARE DISPLAY, EM SEQUÊNCIA VOLUMÉTRICA PONDERADA EM T1 APÓS CONTRASTE.

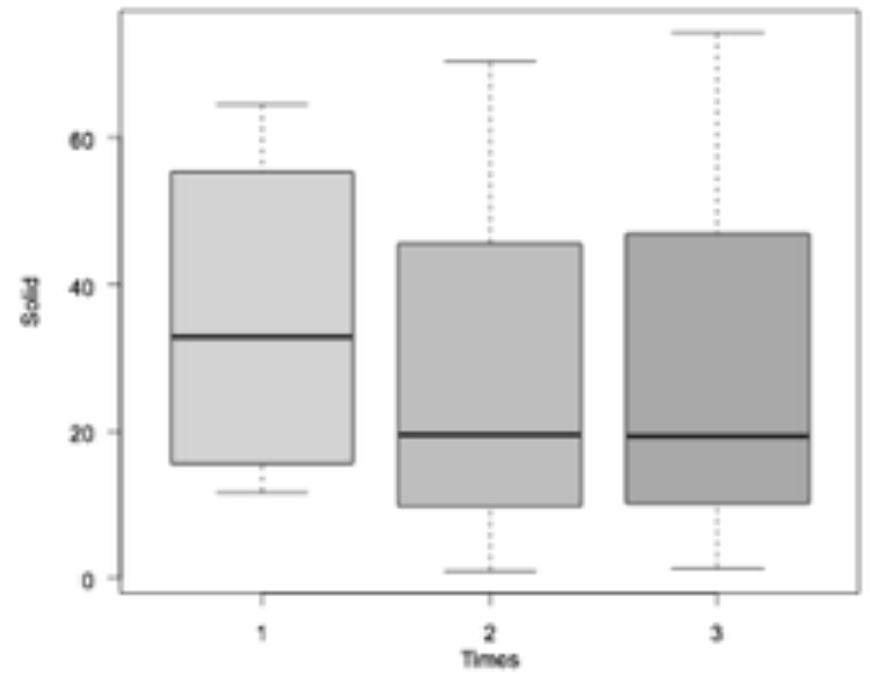

Fig 2: Variação do volume do componente sólido das lesões no intervalo de tempo estudado

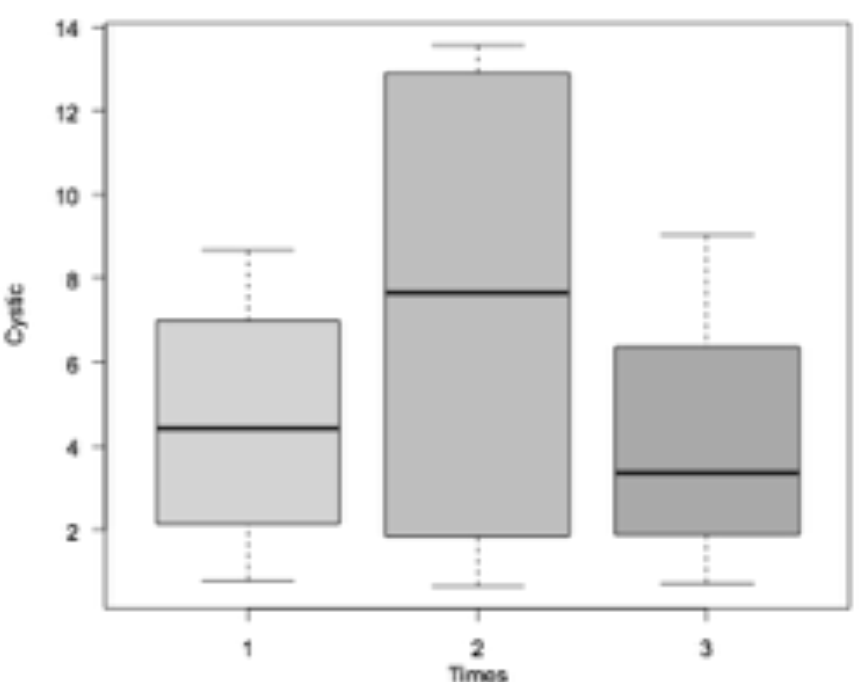

Fig 3: Variação do volume do componente cístico das lesões no intervalo de tempo estudado 


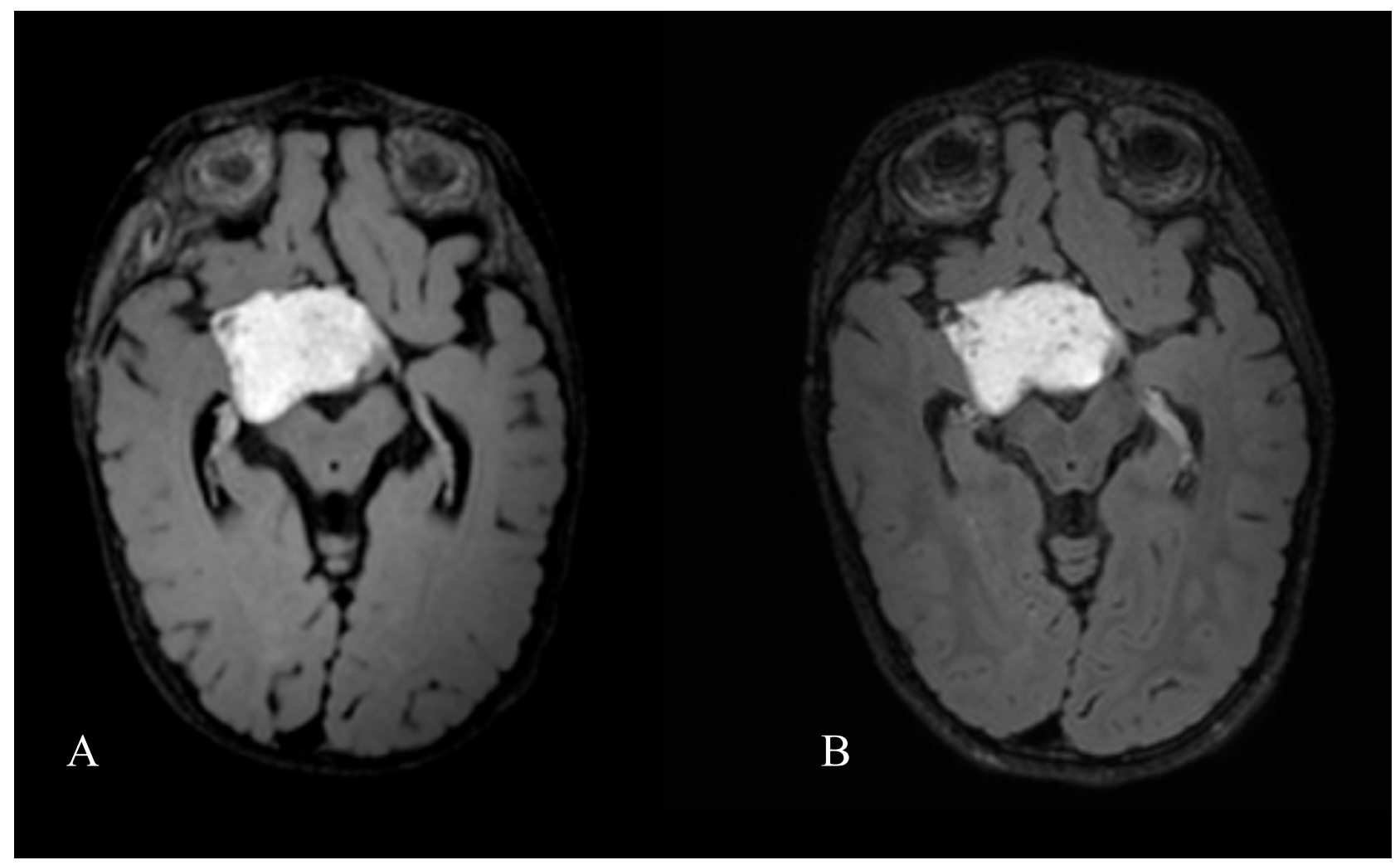

Fig 4: Imagens axiais FLAIR do paciente 6 (Grupo 1) com lesão expansiva quiasmática-hipotalâmica antes da quimioterapia (A) e no seguimento tardio após o tratamento (B), mostram estabilidade das dimensões e aspecto radiológico da lesão

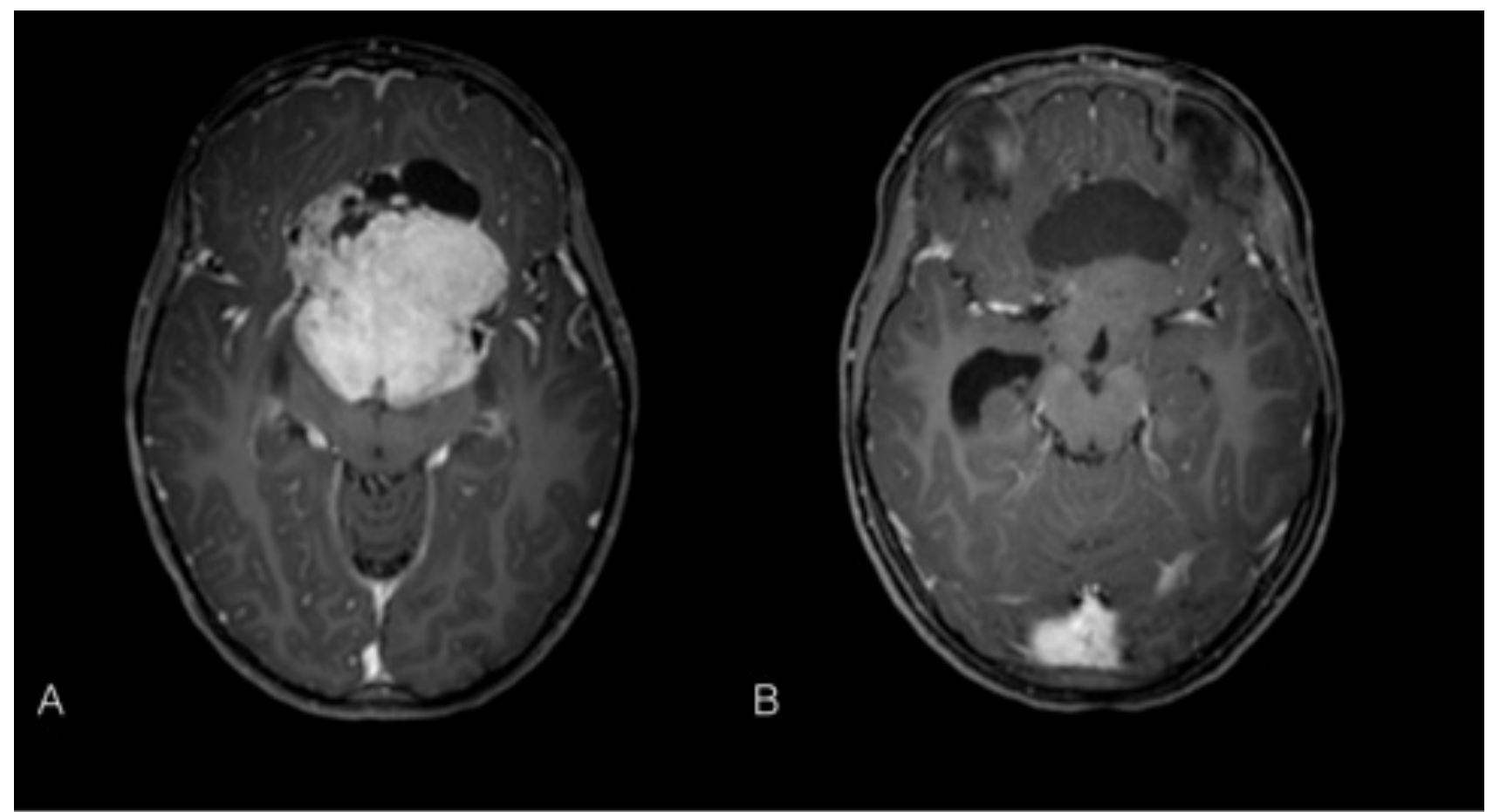

Fig 5: Imagens axiais ponderadas em T1 após administração de contraste do paciente 4 (Grupo 1) com extensa lesão expansiva quiasmática-hipotalâmica antes da quimioterapia (A) e no seguimento tardio após o tratamento (B), com significativa redução do e das áreas de realce da volume da lesão, com aumento do componente cístico 
Group 1 with chemotherapy

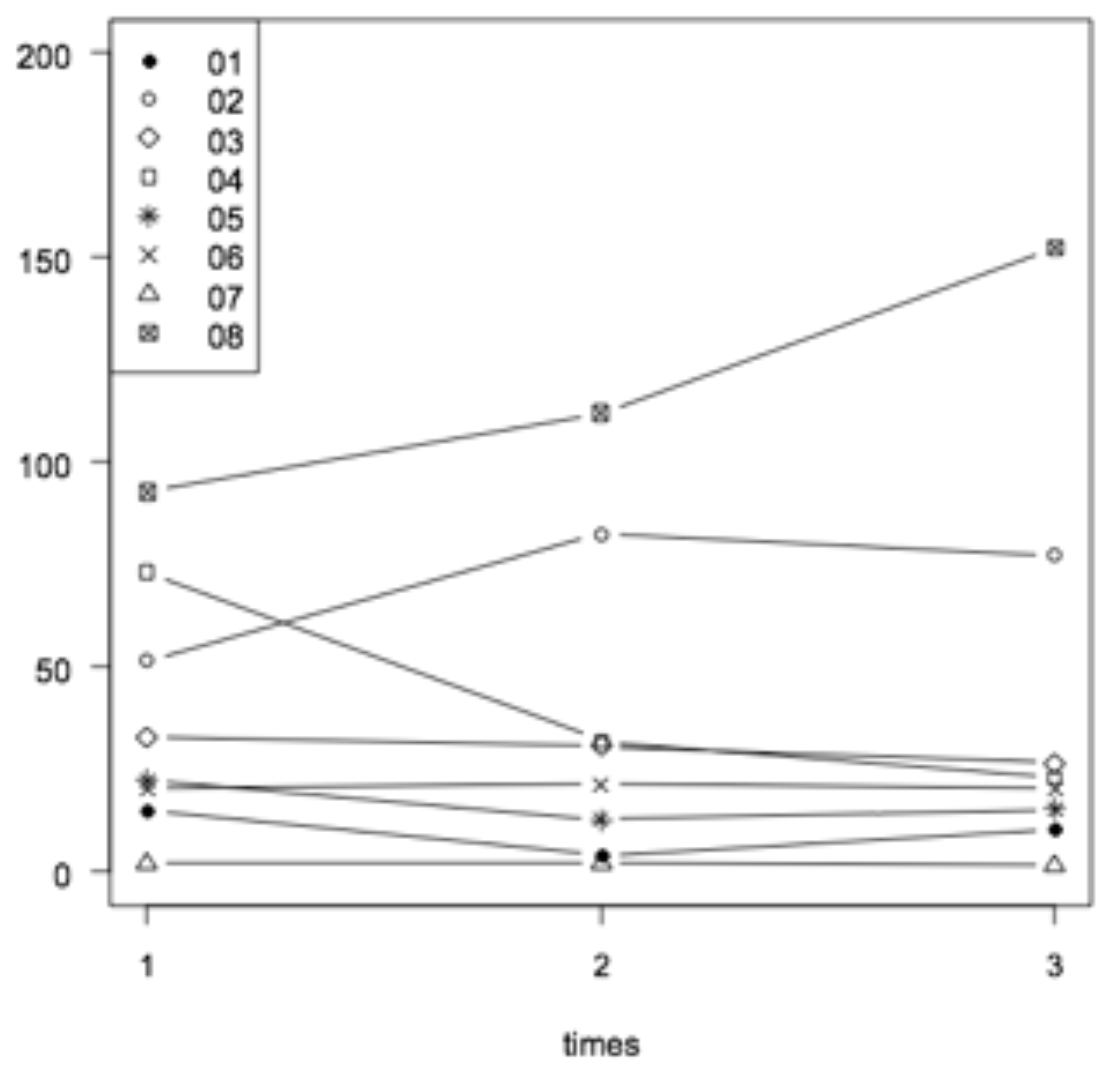

FIG 6: VARIAÇÃO VOLUMÉTRICA DAS LESÕES PARA O GRUPO 1

Group 2 without chemotherapy

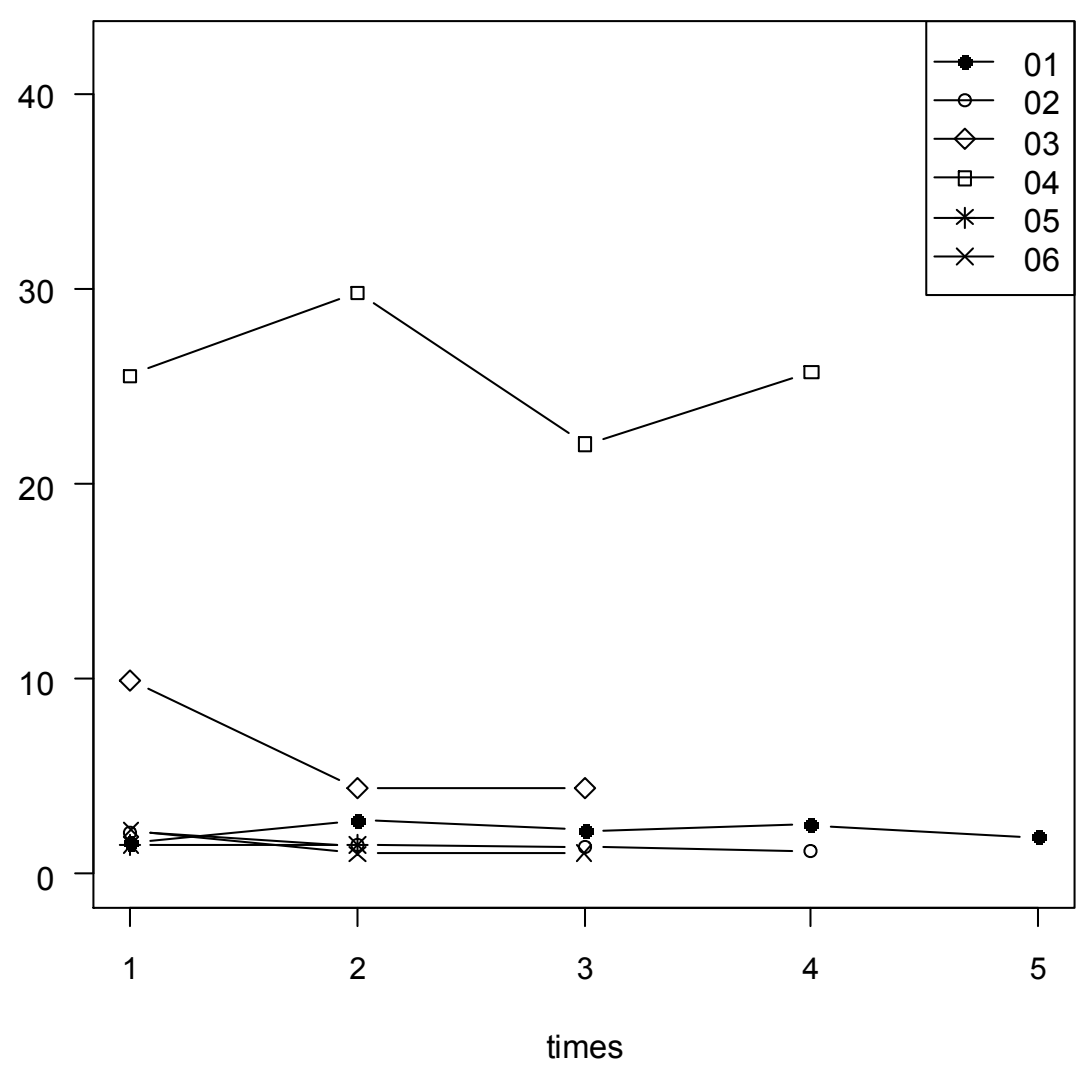

FIG 7: VARIAÇÃO VOLUMÉTRICA DAS LESÕES PARA O GRUPO 2 


\section{Referências}

1. Shamji MF, Benoit BG. Syndromic and sporadic pediatric optic pathway gliomas: Review of clinical and histopathological differences and treatment implications. Neurosurg Focus 2007, 23:E3.

2. Binning MJ, Liu JK, Kestle JR, Brockmeyer DL, Walker ML. Optic pathway gliomas: A review. Neurosurg Focus, $2007,23: E 2$.

3. Kornreich, Liora, et al. Optic pathway glioma: correlation of imaging findings with the presence of neurofibromatosis. American journal of neuroradiology 22.10 (2001): 1963-1969.

4. Dutton, Jonathan J. Gliomas of the anterior visual pathway. Survey of ophthalmology 38.5 (1994): 427-452.

5. Avery, Robert A., Michael J. Fisher, and Grant T. Liu. Optic pathway gliomas. Journal of Neuro-Ophthalmology 31.3 (2011): $269-278$.

6. Czyzyk, Elzbieta, et al. Optic pathway gliomas in children with and without neurofibromatosis 1. Journal of child neurology 18.7 (2003): $471-478$.

7. Danoff, Barbara F., Simon Kramer, and Noble Thompson. The radiotherapeutic management of optic nerve gliomas in children. International Journal of Radiation Oncology Biology Physics 6, n 1, 1980: 45-50.

8. Tao ML, Barnes PD, Billett AL, LeongT, Shrieve DC, Scott RM, Tarbell NJ. Childhood optic chiasm gliomas: radiographic response following radiotherapy and long-term clinical outcome. Int J Radiat Oncol Biol Phys, 1997, 39:579-587.

9. Wisoff JH. Management of optic pathway tumors of childhood. Neurosurg Clin N Am, 1992, 3:791-802.

10. Alvord EC Jr, Lofton S. Gliomas of the optic nerve or chiasm. Outcome by patients age, tumor site and treatmente. J Neurosurg, 1988, 68:85-98.

11. Wisoff JH. Management of optic pathway tumors of childhood. Neurosurg Clin N Am, 1992, 3:791-802.

12 Horwich, A. and Bloom, H.J.G. Optic gliomas: Radiation therapy and prognosis. Int. J. Radiat. Oncol. Biol. Phys, 1985, 11: 1067-1079,

13. Jenkin, D., Angyalfi, S. and Becker, L., et al. Optic glioma in children: Surveillance, resection, or irradiation?. Int. J. Radiat. Oncol, 1993, Biol. Phys. 25: 215-225.

14. Packer, Roger J., et al. Carboplatin and vincristine chemotherapy for children with newly diagnosed progressive low-grade gliomas. Journal of neurosurgery $86, \mathrm{n} 5,1997,747-754$.

15. Erkal, Haldun Şükrü, Meltem Serin, and Ahmet Çakmak. Management of optic pathway and chiasmatic-hypothalamic gliomas in children with radiation therapy. Radiotherapy and oncology 45.1 (1997): 11-15.

16. Packer RJ, Sutton LN, Bilaniuk LT, Radcliffe J, Rosenstock JG, Siegel KR, Bunin GR, Savino PJ, Bruce DA, Schut L. Treatment of chiasmatic/hypothalamic gliomas of childhood with chemotherapy: An update. Ann Neurol 1988, 23:79-85.

17. Grabenbauer, Gerhard G., et al. Radiation therapy of optico-hypothalamic gliomas (OHG)-radiographic response, vision and late toxicity. Radiotherapy and Oncology 54.3 (2000): 239-245.

18. Grill, J., et al. Radiation-induced cerebral vasculopathy in children with neurofibromatosis and optic pathway glioma.Annals of neurology 45.3 (1999): 393-396.

19.Jahraus, Christopher D., and Nancy J. Tarbell. "Optic pathway gliomas." Pediatric blood \& cancer 46.5 (2006): 586-596.

20. Lafay-Cousin L, Holm S, Qaddoumi I, Nicolin G, Bartels U, Tabori U, Huang A, Bouffet E. Weekly vinblastine in pediatric low-grade glioma patients with carboplatin allergic reaction. Cancer 2005, 103:2636-2642.

21. Lancaster DL, Hoddes JA, Michalski A. Tolerance of nitrosurea-based multiagent chemotherapy regime for low-grade pediatric gliomas. J Neuro-oncol 2003;63:289-294.

22. Listernick R, Ferner RE, Liu GT, Gutmann DH. Optic pathway gliomas in neurofibromatosis-1: Controversies and recommendations. Ann Neurol 200, 61:189-198.

23. Grill J, Laithier V, Rodriguez D, Raquin MA, Pierre-Kahn A, Kalifa C. When do children with optic pathway tumours need treatment? An oncological perspective in 106 patients treated in a single centre. Eur J Pediatr 2000, 159:692-696.

24. Chateil JF, Soussotte C, Pedespan JM, Brun M, Le Manh C, Diard F. MRI and clinical differences between optic pathway tumours in children with and without neurofibromatosis. Br J Radiol. 2001;74:24-31

25. Taylor, Timothy, et al. Radiological classification of optic pathway gliomas: experience of a modified functional classification system. The British journal of radiology 2014 
26. Shofty, Ben, et al. Visual outcome following chemotherapy for progressive optic pathway gliomas. Pediatric blood \& cancer 57.3 2011: 481-485.

27. Shofty, Ben, et al. The effect of chemotherapy on optic pathway gliomas and their sub-components: A volumetric MR analysis study. Pediatric blood \& cancer 62.8 (2015): 1353-1359.

28. Opocher, Enrico, et al. Prognostic factors for progression of childhood optic pathway glioma: a systematic review. European journal of cancer 42.12 (2006): 1807-1816

29. Fisher, Michael J., et al. Visual outcomes in children with neurofibromatosis type 1-associated optic pathway glioma following chemotherapy: a multicenter retrospective analysis. Neuro-oncology (2012): nos076.

30. Gnekow, A. K. Recommendations of the Brain Tumor Subcommittee for the reporting of trials. Medical and pediatric oncology 24.2 (1995): 104-108.

31. Moreno, Lucas, et al. Does chemotherapy affect the visual outcome in children with optic pathway glioma? A systematic review of the evidence. European Journal of Cancer 46.12 (2010): 2253-2259.

32.Doganis, Dimitrios, et al. Optic pathway glioma in children: 10 years of experience in a single institution.Pediatric hematology and oncology 33.2 (2016): 102-108. 